\title{
CORRECTION ON A PREVIOUS PAPER
}

BY

E. M. STEIN

The purpose of this note is to correct an error which occurs in the proof of Lemma 5 of [1]. The inequality (3.3) is incorrect and should be replaced by $\left|k_{t}(x)\right| \leqq(A /|x|)(1+\log (|x| t))$, if $t \geqq 1 /|x|$, and $n \geqq 2$. (Hence, in addition, inequality $\left(3.3^{*}\right)$ does not need a separate proof.)

Arguing as before we have,

$$
k_{t}(x)=\int_{\Sigma} \frac{e^{-i r t \cos \left(x^{\prime}, y^{\prime}\right)}-1}{r \cos \left(x^{\prime}, y^{\prime}\right)} \Omega\left(y^{\prime}\right) d \Sigma .
$$

Divide the unit sphere $\Sigma$ into two disjoint regions, $\Sigma_{1}$, and $\Sigma_{\mathbf{2}}$, so that $\Sigma_{1}=\left\{y^{\prime}|| \cos \left(x^{\prime}, y^{\prime}\right) \mid \leqq 1 / r t\right\},(r t \geqq 1)$, and $\Sigma_{2}$ is the complement.

The integral corresponding to $\Sigma_{1}$ is estimated by

$$
A \int_{\Sigma_{1}}\left|\frac{r t \cos \left(x^{\prime}, y^{\prime}\right)}{r \cos \left(x^{\prime}, y^{\prime}\right)}\right| d \Sigma=A t \int_{\Sigma_{1}} d \Sigma=t O(1 / r t)=O(1 / r)
$$

The integral corresponding to $\Sigma_{2}$ is estimated by

$$
A \int_{\Sigma_{2}} \frac{d \Sigma}{r\left|\cos \left(x^{\prime}, y^{\prime}\right)\right|}=\frac{A}{r} \int_{\Sigma_{2}} \frac{d \Sigma}{\left|\cos \left(x^{\prime}, y^{\prime}\right)\right|} \leqq \frac{A}{r}(1+\log (r t)) .
$$

The rest of the proof of the lemma is then concluded as before.

\section{REFERENCE}

1. E. M. Stein, On the functions Littlewood-Paley, Lusin, and Marcinkiewicz, Trans. Amer. Math. Soc. vol. 88 (1958) pp. 430-466. 